\title{
Problem Solving, Computer Technology, and Students' Motivation in Learning Mathematics
}

\author{
Yixun Shi \\ Department of Mathematics, Computer Science and Statistics Bloomsburg University of Pennsylvania Bloomsburg, PA \\ 17815, USA
}

\begin{abstract}
In recent years, more and more attentions are given to developing problem solving skills and using computer technology in the teaching and learning of mathematics. Case studies, independent projects, and examples of applications of mathematics are used more and more frequently in mathematics classes in order to enhance students' development in mathematical thinking and problem solving skills. Two examples of such studies are presented in this paper.
\end{abstract}

Index Terms: Problem solving; Computer applications; Number games; Mathematics education; Student projects

(C) 2011 Published by MECS Publisher. Selection and/or peer review under responsibility of the International Conference on E-Business System and Education Technology

\section{Introduction}

In recent years, mathematics educators are getting more and more aware of the importance of developing problem solving skills and using computer technology in the teaching and learning of mathematics. Case studies, independent projects, and examples of applications of mathematics are used more and more frequently in mathematics classes in order to enhance students' development in mathematical thinking and problem solving skills.

In this paper, I will present two application examples that can be used in teaching various mathematics subjects at college, secondary, or elementary levels. These examples may not only be used to demonstrate or explain mathematics concepts, but will also display various strategies for solving the same problem and explore the connections between different mathematical procedures. Thus they may help students gain a deeper insight of mathematical patterns, and enhance their skills of problem solving. These examples also require the use of computer technology in problem solving.

The examples given in this paper are developed from simple but interesting number games. Since most students tend to enjoy games, examples of this kind will in general raise students' interests and motivation in learning mathematics. In this paper, we will also address the practical ways of using these application examples within a mathematics classroom. The ideas 
and strategies of using games in teaching mathematics are most beneficial to mathematics educators and students.

The following section II presents the application of a number game "24-points". Then in section III the application of the "containers" game is discussed. Some further comments are given in the last section of the paper.

\section{Application Of Number Game 24-Points}

24-points is a simple number game that young kids play to become capable of figuring numbers out right and fast. School teachers have also found it popular because of its effectiveness in improving children's ability in doing mental arithmetic. There exist a variety of different ways to play the game, but essentially they follow these five rules:

1) Four numbers are randomly chosen from the set $\{1,2,3,4,5,6,7,8,9,10\}$ with replacement;

2) Only the operations addition, subtraction, multiplication, and division may be used on the four numbers and intermediate results;

3) All intermediate results must be non-negative integers;

4) Each of the four chosen numbers must be used once and only once;

5) The goal is to get exactly 24.

For example, if the four chosen numbers are 2, 2, 5, 7, then these operations give 24:

$\underline{2} \times \underline{5}=10, \underline{2} \times \underline{7}=14,10+14=24$

Or equivalently, $2 \times 5+2 \times 7=14$. Note that each of the four numbers $2,2,5$, and 7 is used once and only once. Each 2 in the group is considered as an individual number. Also notice that the intermediate results 10 and 14 are both non-negative integers, as required by rule 3 .

Another example also illustrates the rules. If the four chosen numbers are 1, 2, 3, 5, then 24 can be reached by

$\underline{5}+\underline{1}=6, \underline{2}+6=8,8 \times \underline{3}=24$.

Here, 24 is given by $(5+1+2) \times 3$. Again, each of the four numbers is used exactly once, and the intermediate results 6 and 8 are non-negative integers.

The arithmetic combinations used in the two examples represent two different types of procedures. In the first example, the four numbers are paired. Arithmetic operations are applied to each pair and then on the resulting numbers. That represents the basic concept of parallel computation. In the second example, the arithmetic operations are applied to two of the numbers, then the third number, and finally the last number. That represents the basic concept of sequential computation. In the classroom, the teacher should mention to students that these two are the only types of procedures involved in this game. This is an important fact to be used by students when they write computer programs or use computer software to solve the game.

This game is most conveniently played with a deck of 40 playing cards. The face-cards and the two jokers are not used. Only number-cards (Ace used as 1) are used. Four cards are picked at random from the deck. Because the deck contained four of each number in $\{1,2,3,4,5,6,7,8,9,10\}$, a single number can appear up to four times.

Students should be aware that given four numbers there may be a few alternative ways to get 24 . They should also be reminded that some combination of four numbers will never yield 24 . For example, if the four numbers are $2,2,2,2$, then the number 24 cannot be obtained under the rules of the game.

Following questions now can be asked and studied in the classroom:

1) How many possible ways are there to apply arithmetic operations on the four numbers from the cards?

2) What is the probability of getting a set of four cards for which there is a way to get 24 (such a set may be called a doable set)?

3) Why is 24 chosen as the targeted number? Why not 23 or 25 ? Are there "better" choices for the targeted number than 24 ? And what is meant by "better"?

These questions can be given to students as case studies or independent projects, or used as study examples in class. One possible way of defining "better" is to compare the probability of getting a doable set (the larger this probability the better) 
and also to examine number of alternative ways of getting 24 from a doable set (the more the better). Based on this definition, the number 24 does seem better than a few neighboring numbers, as shown by the following table from [3]. See [3] for a detailed analysis yielding results given in this table.

Table 1. Comparison of some targeted numbers

\begin{tabular}{|c|c|c|}
\hline targeted number & $\begin{array}{c}\text { probability of getting a } \\
\text { doable set }\end{array}$ & $\begin{array}{c}\text { Average number of alternative ways to } \\
\text { get the targeted number }\end{array}$ \\
\hline 22 & $75.92 \%$ & 6.4 \\
\hline 23 & $69.91 \%$ & 5.6 \\
\hline 24 & $86.35 \%$ & 5.8 \\
\hline 25 & $68.81 \%$ & 4.7 \\
\hline 26 & $73.05 \%$ & \\
\hline
\end{tabular}

The study of this example will involve use of counting rules, probability theory, and computer technology. It is most appropriate to be used at high school or college probability/statistics classes. Students should also be asked to consider these questions:

1) If we use a computer to randomly generate four integers from the set $\{1,2,3,4,5,6,7,8,9$, 10 $\}$, would above results in Table 1 change? If so, how would they change?

2) If we use five numbers instead of four to play the game, what results would we get for Table 1? What if we use six numbers, seven numbers, ...?

\section{Applications of the "Containers" Game}

This game is often used in elementary schools for children to "work out" with numbers through guesses and trials. However, it also provides an interesting example in teaching algebra and number theory. Here is an example of the "containers" game.

"Suppose you have an unlimited amount of apple cider in a large tank. You want to measure 2 gallons of apple cider for a customer. You only have a 4-gallon and 5-gallon measuring device. How could you measure 2 gallons?"

For elementary students, a first approach in solving this problem might be to construct a simple table and begin a trial and error approach under the guidance of the instructor. One possible outcome could be as given in Table 2.

From this table we see that 2 gallons of apple cider is measured from a 4-gallon and a 5-gallon container. Note that this table demonstrates only one possible solution.

Teachers often would ask students to find a few alternative procedures to get the measuring, also using the guess and trial method.

Also ask students to observe the total number of actions used in each alternative measuring procedure. With which procedure would we use the least amount of actions? 
Table 2. Measuring 2 gallons of apple cider

\begin{tabular}{|c|c|c|}
\hline Action & $\begin{array}{c}\text { Amount in the 4 } \\
\text { gallon container } \\
\text { (A) }\end{array}$ & $\begin{array}{c}\text { Amount in the 5 } \\
\text { gallon container } \\
\text { (B) }\end{array}$ \\
\hline Fill (B) & 0 & 5 \\
\hline $\begin{array}{c}\text { Pour cider from (B) to } \\
\text { (A) until (A) is full }\end{array}$ & 4 & 1 \\
\hline Empty (A) & 0 & 1 \\
\hline $\begin{array}{c}\text { Pour the cider remaining } \\
\text { in (B) to (A) }\end{array}$ & 1 & 0 \\
\hline Fill (B) & 1 & 2 \\
\hline $\begin{array}{c}\text { Pour the cider from (B) to } \\
\text { (A) until (A) is full }\end{array}$ & 4 & 2 \\
\hline Empty (A) & 0 & \\
\hline
\end{tabular}

For college algebra or number theory students, a closer look at this problem reveals that there are only 3 different actions involved in this procedure: (1) filling a container; (2) transferring from one container to another; and (3) emptying a container. Moreover, suppose we use $\mathrm{Q}$ to denote the total quantity of cider we have in the containers, then before any action is taken the value of Q equals zero. During the procedure, each action (1) adds 4 or 5 to Q, each action (3) subtracts 4 or 5 from Q, and action (2) does not change the value of $\mathrm{Q}$. Our goal in this procedure is to make $\mathrm{Q}=2$ through a sequence of actions. Also note that at any time during this procedure the value of $Q$ can never go beyond 9 , the sum of 4 and 5 .

With that in mind, we see that the above actions form a string of "+" and " $-":+5-4+5-4=2$. This string, of course, comes with the caveat that we can never have more than 9 gallons ( 4 gallon +5 gallon) in total at any given time, or else both containers would be over-full and thus not able to be measured. Therefore, the string $+5+5-4-4$ cannot be used in this case. In other words, although both $+5-4+5-4$ and $+5+5-4-4$ equals $5(2)-4(2)=2$, the first string represents a sequence of actions to solve the problem while the second string does not.

As we progress with this problem, we may mention to students that, in order to find out an appropriate string of operations, we first need to find two integer numbers $a$ and $b$ such that

$2=4 a+5 b$

This may be equivalently expressed as to find two positive integers $\mathrm{n}$ and $\mathrm{m}$ such that either

$2=4 n-5 m \quad$ or $\quad 2=5 m-4 n$.

Once such a combination is found, a string of arithmetic operations can be set up correspondingly. For example, the string $+5-4+5-4=2$ is based on the combination $2=5(2)-4(2)$. In this case, the combination is of the form $2=5 \mathrm{~m}-4 \mathrm{n}$, with $\mathrm{m}=\mathrm{n}=2$.

The following questions can now be asked and studied by students with proper guidance of the teacher:

1) Given an $x$-gallon container and a $y$-gallon container, can we measure $\mathrm{S}$ gallons for any integer $\mathrm{S} \leq x+y$ ? (Answer: "no" in general, "yes" if $x$ and $y$ are relatively prime. See [2] for details.)

2) What is the necessary and sufficient condition for us to be able to measure $\mathrm{S}$ gallons using an $x$-gallon container and a $y$-gallon container? (Answer: $\mathrm{S}$ is a multiple of the largest common factor of $x$ and $y$ ). 
3) When $\mathrm{S}$ gallons can be measured using an $x$-gallon container and a $y$-gallon container, how would we mathematically find two integers $a$ and $b$ such that $S=a x+b y$ without guessing and trialing? (Answer: use the facts about linear Diophantine equation. See [1] for details)

4) Continue with question 3 , what is the minimum total number of actions needed to measure $S$ gallons based on the equation $\mathrm{S}=\mathrm{a} x+\mathrm{by}$ ? $($ Answer: $2(|\mathrm{a}|+|\mathrm{b}|)-1)$

5) Continue with question 4 , how would we write a computer program or use a computer software to find two integers $\mathrm{a}$ and $\mathrm{b}$ such that $\mathrm{S}=\mathrm{a} x+\mathrm{by}$ and meanwhile make the value $|\mathrm{a}|+|\mathrm{b}|$ as small as possible? That is, to solve the problem minimize $|a|+|b|$ subject to: $S=a x+b y$ a and $b$ are integers

6) Continue with question 5 , how would we write a computer program or use a computer software to construct a proper string of actions based on the equation $\mathrm{S}=\mathrm{a} x+\mathrm{b} y$ to measure $\mathrm{S}$ gallons?

For answers to questions 5 and 6, see [1] and [2]. This study involves the use of concepts of relatively prime numbers, combinations of numbers, linear Diophantine equations, optimal solution, algorithmic procedures, and computer applications. Further, we may ask students to consider the case when more than two containers are used to do the measuring. More precisely, assuming that we are given $\mathrm{k}$ containers of capacity $\mathrm{a}_{1}, \mathrm{a}_{2}, \ldots \mathrm{a}_{\mathrm{k}}$ gallons, also assuming that the largest common factor of $a_{1}, a_{2}, \ldots a_{k}$ is 1 , we want to measure $c$ gallons with $c$ being less than or equal to $a_{1}+a_{2}+\ldots+a_{k}$. This can be an interesting and beneficial case study for students to conduct.

\section{Further Comments}

Developing problem solving skills and using computer technology in mathematics education have proven to be effective in motivating students in the learning process. Study of games will also raise students' interests in mathematics. In general, one may seek to mathematically analyze various sport games, puzzles, number games, and so on to provide mathematics teachers and students more interesting application examples which usually also involve the use of computer technology. The significance of these studies is to help students see how various mathematical concepts and methods can be applied together in studying the same object. This would help students to gain a deeper insight into connections between different mathematics concepts. They will also help students to link mathematics to the real life, and therefore promote their interests in mathematics and help them to better understand mathematics concepts. These activities will also help students enhance mathematical thinking and develop problem solving skills.

\section{References}

[1] E. Mauch and Y. Shi, "Application of linear Diophantine equation in teaching mathematical thinking", Mathematics and Computer Education, 37(2), pp 240 -- 247, 2003

[2] E. Mauch and Y. Shi, "One approach to explore a range of problem solving strategies in the classroom", International Journal of Mathematical Education in Science and Technology, 35 (6), pp 912 -- 917, 2004.

[3] Y. Shi, "A mathematical study of the game Twenty Four Points", Mathematics Teacher, 92(9), pp 828 -- 832, 1999. 